\title{
Magnetic Adatom Induced Skyrmion-Like Spin Texture in Surface Electron Waves
}

\author{
Samir Lounis, ${ }^{*}$ Andreas Bringer, and Stefan Blügel \\ Peter Grünberg Institut and Institute for Advanced Simulation, Forschungszentrum Jülich and JARA, D-52425 Jülich, Germany
} (Received 20 December 2011; published 14 May 2012)

\begin{abstract}
When a foreign atom is placed on a surface of a metal, the surrounding sea of electrons responds by screening the additional charge leading to oscillations or ripples. On surfaces, those electrons are sometimes confined to two-dimensional surface states, whose spin-degeneracy is lifted due to the Rashba effect arising from the spin-orbit interaction of electrons and the inversion asymmetric environment. It is believed that at least for a single adatom scanning tunneling microscopy measurements are insensitive to the Rashba splitting; i.e., no signatures in the charge oscillations will be observed. Resting on scattering theory, we demonstrate that, if magnetic, one single adatom is enough to visualize the presence of the Rashba effect in terms of an induced spin magnetization of the surrounding electrons exhibiting a twisted spin texture described as superposition of two Skyrmionic waves of opposite chirality.
\end{abstract}

The lack of spatial inversion symmetry is the triggering ingredient for a wide range of new phenomena that are accessible with state-of-the-art experimental techniques [1-6]. Angle-resolved photoemission spectroscopy was the first tool used by LaShell and co-workers [7] to discover a small energy splitting in the $s p$ surface state band of the $\mathrm{Au}(111)$ surface. This splitting has been interpreted by the same authors as a realization of the interaction between the spin and orbital angular momentum, which promoted the renaissance of the Rashba physics. The surge of interest in similar effects involving the spin-orbit interaction in a structural asymmetric environment is the incitement of many additional sophisticated measurements and theoretical simulations on relevant surfaces with [8-10] and without [5,11-15] topologically protected surface states. Since charge oscillations (Friedel oscillations) induced by scattering of the surface-state electrons at a single adatom are blind with respect to such effects [16], probing with the scanning tunneling microscope (STM) charge oscillations confined within a corral of adatoms has been proposed on the basis of a multiple scattering study as an alternative [17]. Theoretical calculations [18] outline the possibility of very complex magnetic structures. We pursue a different route and investigate the possibility of grasping information on the spin-orbit interaction at surfaces exploiting the break of time inversion symmetry introduced by a magnetic adatom. As shown and discussed later, we discover an intriguing spin texture in the spin-polarized electron gas surrounding the magnetic adatom (Fig. 1). This new magnetic behavior can be very similar to the topological twists, called Skyrmions [1-3,19,20]. Parts of our predictions have recently been verified in the interferences produced by the scattering at a $\mathrm{MnPc}$ molecule of the complex surface states of $\mathrm{Bi}(110)$ surface [15].

Our investigation is based on a Rashba Hamiltonian [21] that describes a two-dimensional gas of free electrons confined in the $(x y)$ plane of a metallic surface:

$$
\hat{H}=\frac{\hat{p}_{x}^{2}+\hat{p}_{y}^{2}}{2 m^{*}}-\frac{\alpha_{R}}{\hbar}\left(\sigma_{x} \hat{p}_{y}-\sigma_{y} \hat{p}_{x}\right)
$$

is considered with respect to a zero-energy reference defined by the bottom of the dispersion curve in the absence of the spin-orbit coupling. In this equation, $m^{*}$ is the effective mass of the electron and $\alpha_{R}$ is the effective Rashba parameter, describing the strength of the effect, whose value is determined in principle by the atomic spinorbit strength as well as by the degree of asymmetry of the wave function imposed by the presence of the surface [16].

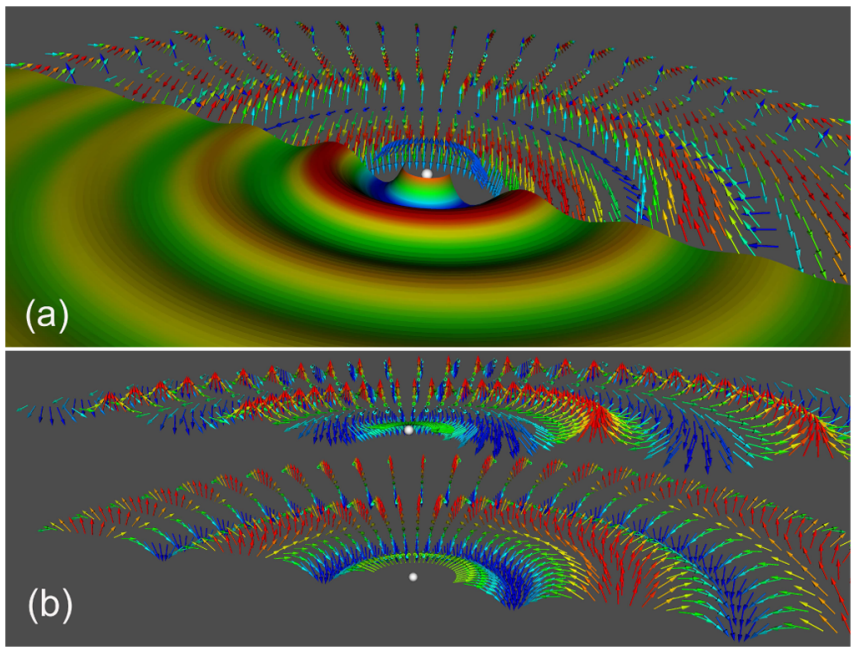

FIG. 1 (color online). Skyrmionic-like spin texture at the Fermi energy of Au(111) surface. (a) Spatial visualization of the induced local density of states (LDOS) and the local magnetization direction of Au surface electrons after scattering with a Fe adatom. The spin texture found in (a) can be decomposed into a linear combination of two smoothly rotating Skyrmionic magnetic waves with opposite vector chirality shown in (b). The wavelengths of LDOS oscillation, the left and right Skyrmionic waves are $\sim 18.7, \sim 17.3$, and $20.3 \AA$, respectively. 
Here, however, $\alpha_{R}$ is chosen to model the experimental dispersion relation of the surface state. Quite generally the gradient of the potential at the surface acts as an electric field $\vec{E}$ normal to the surface in the lab frame of the sample. Electrons propagating with momentum $\vec{k}$ across the surface experience this field in their local frame of reference as an effective magnetic field, $\vec{B}=\frac{\hbar}{m^{*} c} \vec{k} \times \vec{E}$, which is the origin of the functional form for the Rashba Hamiltonian and defines a spin-quantization axis $\hat{n}(\vec{k})$ to be located in the surface plane normal to the wave vector $\vec{k}=\left(k_{x}, k_{y}\right)=$ $k(\cos \phi, \sin \phi) \perp \hat{n}(\vec{k})$, and $c$ is the speed of light. The eigenvectors $\left|\psi_{1(2)}\right\rangle$ of the Hamiltonian (1), associated with the wave vectors $\vec{k}_{1}$ and $\vec{k}_{2}$, are spin-up and spindown states, respectively, with respect to $\hat{n}$, but are also a coherent superposition of spin-up and spin-down states, $\left|\psi_{1(2)}(\vec{k})\right\rangle=\frac{e^{i k_{1(2)}}}{\sqrt{2}}\left(|\uparrow\rangle-(+) i e^{i \phi}|\downarrow\rangle\right)$, expressed by the spin functions $|\uparrow\rangle$ and $|\downarrow\rangle$, when measured with respect to the surface normal ( $z$ direction). The eigenvalue spectrum $E_{1(2)}(\vec{k})=\frac{\hbar^{2}}{2 m^{*}}\left(k_{1(2)}^{2}-k_{\mathrm{so}}^{2}\right)$ is a two spin-split cone-shaped parabolic energy dispersion curve by which the origins of the parabola $E_{1(2)}$ are shifted with respect to $k=0$ by $k_{\text {so }}= \pm m^{*} \alpha_{R} / \hbar^{2}$, i.e., $k_{1(2)}=k+(-) k_{\text {so }}$ (see Fig. 2). When $k_{2}$ changes sign from positive to negative, the two branches of the dispersion curves cross. Unless stated otherwise, we shall consider only positive $k_{2}$ in the upcoming text.

The scattering of the surface-state Rashba electrons on a magnetic adatom deposited on a substrate is investigated using scattering theory, which has been successfully applied in the description of electron scattering at adatoms [22-24]. This theory involves the calculation of Green functions, which allow an elegant treatment of the elec-

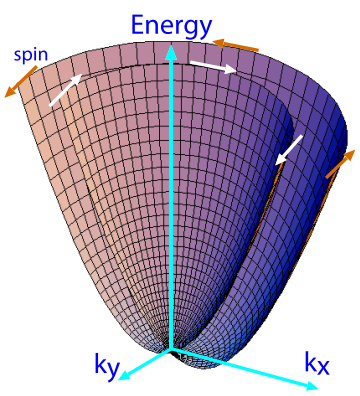

FIG. 2 (color online). Energy dispersion of spin-split surfacestate electrons. When plotted with respect to the two components of the two-dimensional wave vector $\vec{k}$, the energy dispersion is cone shaped. Because of spin-orbit interaction, two spin-split parabolas are obtained and are centered around $\pm k_{\mathrm{so}}$ (see text). Arrows indicate the vector fields of the spin-quantization axes (or the patterns of the spin) at the constant energy contour. For every energy, two opposite spins have different wave vectors leading to two concentric circles with opposite spins. The effective $B$ field felt by the electrons is perpendicular to the propagation direction defined by $\vec{k}$. tronic properties including the embedded adatom by solving the Dyson equation, $G=G_{0}+G_{0} t G_{0}$, where all quantities are site, spin, and energy dependent matrices. We note that the electronic properties of the adatom are inscribed into the scattering matrix $t$, the amplitude of the electron wave scattering at the adatom. $G_{0}$ is the Green function of the pure two-dimensional electron gas corresponding to the Hamiltonian (1) that is constructed using the eigenvectors $\left|\psi_{1(2)}\right\rangle$ :

$$
G_{0}=\left[\begin{array}{cc}
G_{\mathrm{D}} & e^{-i \phi} G_{\mathrm{ND}} \\
-e^{i \phi} G_{\mathrm{ND}} & G_{\mathrm{D}}
\end{array}\right] .
$$

In case the Rashba effect vanishes, the off-diagonal part of the Green function, $G_{\mathrm{ND}}$, is zero and the diagonal part, $G_{\mathrm{D}}$, reverts to the Green function of the free electron surface states. From $G$, quantities related to those measured by STM, such as the local density of states (LDOS) [25] $n(\vec{r} ; E)=-1 / \pi \operatorname{Im} \operatorname{Tr}_{\sigma} G(\vec{r}, \vec{r} ; E)$ or measured by the spin-polarized STM (SP STM) as the local magnetization density of states (LMDOS) [26] $\vec{M}(\vec{r} ; E)=$ $-1 / \pi \operatorname{Im} \operatorname{Tr}_{\sigma} \vec{\sigma} G(\vec{r}, \vec{r} ; E)$ can be calculated, where $\vec{\sigma}$ is the vector of Pauli matrices and $\operatorname{Tr}_{\sigma}$ is a trace in spin space. It is convenient to express all quantities in cylindrical coordinates as it turns out that they depend only on the radial distance $R$ measured from the position of the adatom. The LMDOS is expressed by two components, $\vec{M}(R)=\left(M_{z}, M_{r}\right)$, the radial component, $M_{r}$, and the one normal to the surface, $M_{z}$. The azimuthal component, $M_{\phi}$, vanishes.

The obtained results are general, but to strengthen our point we consider the system of a $\mathrm{Fe}$ adatom on the $\mathrm{Au}(111)$ surface as an application, because the system has been proven experimentally accessible by lowtemperature STM and for Au the presence of a large Rashba effect has been shown experimentally [7]. On such a surface, Jamneala and co-workers [27] found that contrary to $\mathrm{Ti}, \mathrm{Co}$, and $\mathrm{Ni}$, no Kondo peak was observed for $\mathrm{V}, \mathrm{Cr}, \mathrm{Mn}$, and $\mathrm{Fe}$ adatoms, which means that for the latter elements a local magnetic moment indeed exists and it is not screened by the conduction electrons [28]. We treat Fe as an adatom with a magnetic moment pointing along the $z$ direction perpendicular to the surface because our firstprinciples calculations [29] (see Supplemental Material [30]) predict this to be the easy axis with a magnetic anisotropy energy of about $12 \mathrm{meV}$.

Hence, such a case imposes a scattering matrix that is diagonal in spin space,

$$
t=\left[\begin{array}{cc}
t_{\uparrow} & 0 \\
0 & t_{\downarrow \downarrow}
\end{array}\right],
$$

which can be related to the phase shift $\delta(E)$ experienced by the incoming electron waves scattering at the adatom $(t=$ $\left.\hbar^{2} / m^{*}[\exp (2 i \delta(E))-1]\right)$. Note that a generalization to an arbitrary rotation angle of the magnetic moment will be straightforward using standard unitary transformations. 
For $\mathrm{Fe}$, as for $\mathrm{Au}$, all majority-spin states are fully occupied and the scattering of majority electrons in the vicinity of the Fermi energy $\left(E_{F}\right)$ is practically zero; i.e., the phase shift vanishes $\left(t_{\uparrow \uparrow}=0\right)$. The minority-spin LDOS shows, on the contrary to $\mathrm{Au}$, high values around the Fermi energy and therefore a large scattering. Thus, we assume a phase shift of $\pi / 2\left(t_{\downarrow \downarrow}=-2 \hbar^{2} / \mathrm{m}^{*}\right)$.

The parameters describing the $\mathrm{Au}(111)$ surface state are identical to those chosen by Walls and Heller [17]: $m^{*}=$ $0.26 m_{e}, E_{F}=0.41 \mathrm{eV}$, and $\alpha_{R}=0.4 \mathrm{eV} \AA$ that correspond to a Fermi wavelength $\lambda_{\mathrm{F}}=\frac{2 \pi}{k} \sim 37.4 \AA$ and a spin-rotation length $\lambda_{\mathrm{so}}=\frac{\pi}{k_{\mathrm{so}}}$ of 230.5 A.

After solving the Dyson equation, the induced circular LDOS oscillations, $\Delta n(R ; E)$, emanating from the adatom located at the center $(R=0)$ are given by $\Delta n=$ $-1 / \pi \operatorname{Im}\left[\left(G_{\mathrm{D}} G_{\mathrm{D}}+G_{\mathrm{ND}} G_{\mathrm{ND}}\right)\left(t_{\Uparrow}+t_{\Downarrow \downarrow}\right)\right]$ and are plotted in Fig. 1(a) for all states at $E=E_{F}$. At large distances $R$, the circular induced standing wave undulations, $\Delta n(R ; E)$, can be expressed as $\sim \frac{m^{*}}{\pi^{2} \hbar^{2} k^{2} R} \sqrt{k_{1} k_{2}} \cos (2 k R)$. When the spin-orbit coupling is negligible, i.e., $k_{1} \sim k_{2}$, we recover the conventional form of the adatom-induced energydependent charge density oscillations $\sim \frac{\cos (2 k R)}{2 k R}[22,23]$. Regardless of the spin-orbit interaction, there is only one wave vector, $2 k=k_{1}+k_{2}$, that describes the oscillations, with the corresponding wavelength at $E_{F}$ given by $\lambda_{F} / 2 \sim$ 18.7 A. Consistent to the work of Petersen and Hedegård [16] and confirmed by Walls and Heller [17], no signature or information on the Rashba effect or spin-orbit interaction of the surface atoms can be extracted from the LDOS.

This picture changes fundamentally when we look at the LMDOS. Because of the presence of a magnetic adatom $\left(t_{\uparrow} \neq t_{\Downarrow \downarrow}\right)$, the magnetization density perpendicu- lar to the surface and in the surface plane is nonzero. We find concentric rings $M(R)$ of equal size magnetization density surrounding the $\mathrm{Fe}$ adatom, with magnetization densities emanated at the center of the Fe atom wrangling in the $\left(M_{z}(R), M_{r}(R)\right)$ plane [Fig. 1(a)]. Contrary to the LDOS, the magnetization density is nontrivially modulated by the Rashba effect. Analyzing the asymptotic behavior of $M_{z}(R) \sim \frac{m^{*}}{2 \pi^{2} \hbar^{2} k^{2} R}\left(k_{1} \cos \left(2 k_{1} R\right)+k_{2} \cos \left(2 k_{2} R\right)\right)$ as calculated from $M_{z}=-1 / \pi \operatorname{Im}\left[\left(G_{\mathrm{D}} G_{\mathrm{D}}-G_{\mathrm{ND}} G_{\mathrm{ND}}\right)\left(t_{\uparrow}-t_{\downarrow \downarrow}\right)\right]$ as well as of $M_{r}(R) \sim \frac{m^{*}}{2 \pi^{2} \hbar^{2} k^{2} R}\left(k_{1} \sin \left(2 k_{1} R\right)-k_{2} \sin \left(2 k_{2} R\right)\right.$ ) as calculated from $M_{r}(R)=-2 / \pi \operatorname{Im}\left[G_{\mathrm{D}}\left(t_{\uparrow \uparrow}-t_{\downarrow \downarrow}\right) G_{\mathrm{ND}}\right]$, one finds that both wave vectors $k_{1}$ and $k_{2}$ enter nontrivially. This observation implies the possibility of using a SP STM with an out-of-plane magnetized tip to probe the $z$-projected Rashba-induced interferences. Any magnetic signal detected by a SP STM with an in-plane magnetized tip would be a clear fingerprint of the Rashba effect. If there is no Rashba effect, i.e., $G_{\mathrm{ND}}=0$, the quantization axis is only determined by the adatom and the scattering electrons are not a coherent superposition of spin-up and spin-down electrons that led to a finite in-plane spin component.

Such spin textures are a reminder of Skyrmionic magnetic configurations but are completely different. Indeed, with Skyrmions, the magnetic moments can rotate smoothly from one direction at the center of the structure to the opposite direction. Here, however, the magnetization ripples experience phase switch as well as beating as sketched in the top right of Fig. 3. In order to simplify our analysis, the induced LDOS and the $z$ and radial components of magnetization passing by the adatom position are additionally illustrated in the top left of the same figure. Surprisingly, the amplitude of the $M_{r}$ and $M_{z}$ oscillations is of the same size with a phase shift between

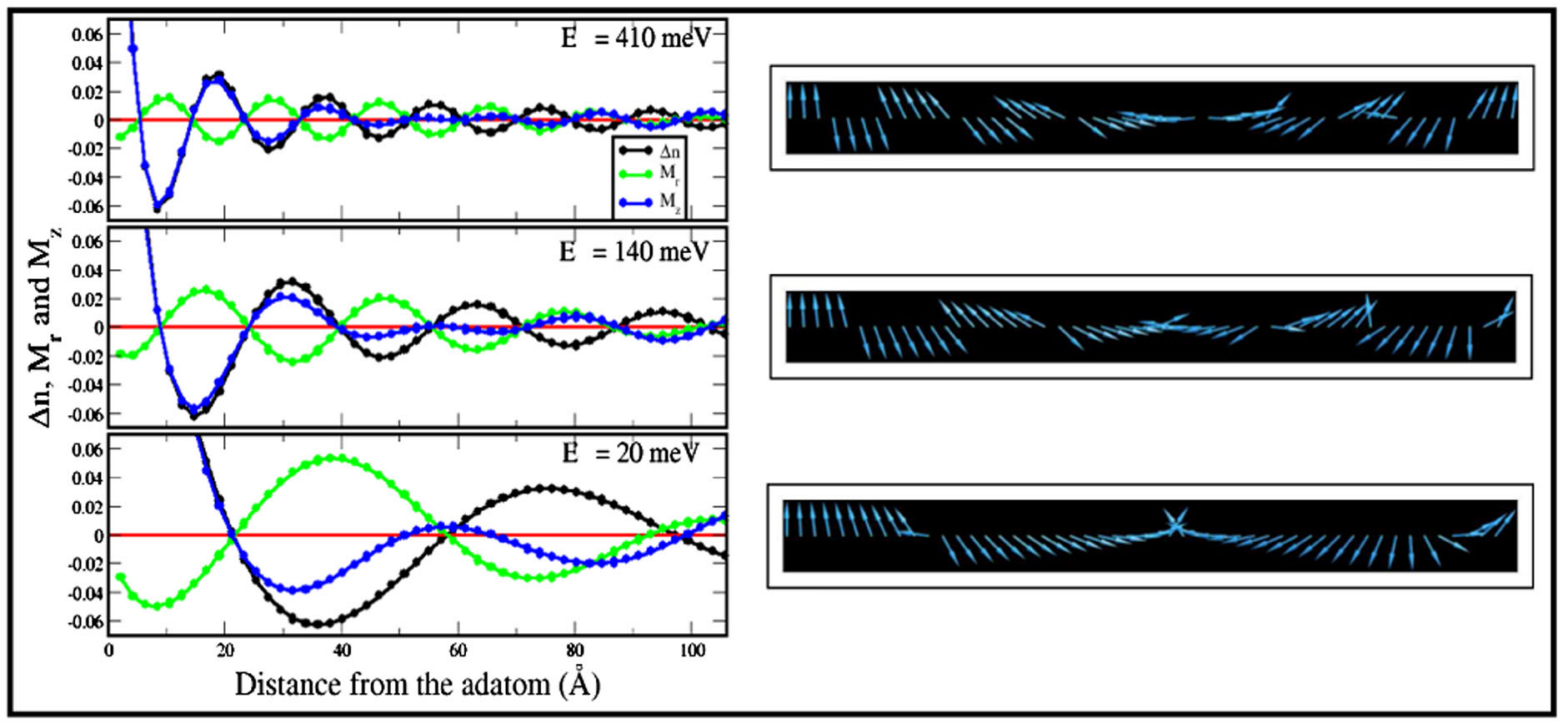

FIG. 3 (color online). Comparison of the induced LDOS and LMDOS components. Because of the Rashba effect the magnetic Fe adatom induces nontrivial spin interferences: The left panels show the radial dependence of the LDOS and LMDOS at different energies, while the right panels depict the corresponding magnetization unit vectors. 
them that depends on the strength of the Rashba coupling term. This can be better understood when looking at the asymptotic behavior. For instance, $M_{z}$ can be rewritten in terms of $k_{\mathrm{so}}$ and $k$ as $\sim \frac{m^{*}}{\pi^{2} \hbar^{2} k^{2} R}\left(k \cos (2 k R) \cos \left(2 k_{\mathrm{so}} R\right)-\right.$ $\left.k_{\mathrm{so}} \sin (2 k R) \sin \left(2 k_{\mathrm{so}} R\right)\right)$. At the Fermi energy of gold, $k_{\mathrm{so}}$ is expected to be very small compared to $k$. At distances much smaller than the spin-rotation length but long enough that the previous asymptotic behavior holds, $M_{z}$ simplifies more to $\frac{m^{*}}{\pi^{2} \hbar^{2} k R} \cos (2 k R)$ and oscillates similarly to the LDOS with or without spin-orbit interaction. This explains the wavelengths of the induced initial ripples being, as expected, twice the Fermi wave vector. In fact, one has to go far beyond the vicinity of the adatom, at distances of the order of the spin-rotation length, to see an effect achieved by the intriguing phase switch observed around $60 \AA$. Indeed, the new spin-rotation length corresponding to a full rotation from $0^{\circ}$ to $180^{\circ}$ occurs around $115 \AA$, which means that the change of sign occurs at half this value. Another substrate with a stronger Rashba coupling parameter would impose a more important phase shift at smaller distances. $M_{r}$, which is proportional to $-\frac{m^{*}}{\pi^{2} \hbar^{2} k^{2} R}\left(k \cos (2 k R) \sin \left(2 k_{\mathrm{so}} R\right)+k_{\mathrm{so}} \sin 2 k R \cos \left(2 k_{\mathrm{so}} R\right)\right)$ can be further simplified-for rather short distances-to $-\frac{2 m^{*} k_{\text {so }}}{\pi^{2} \hbar^{2} k} \cos (2 k R)$, similar to the cosine behavior that characterizes $M_{z}$.

Although the spin texture looks very complex at first sight, it can be understood as a linear combination of two Skyrmionic waves, $\vec{M}=\vec{M}_{k_{1}}+\vec{M}_{k_{2}}$, with $\vec{M}_{k_{1}}(R) \propto$ $k_{1}\left(\cos \left(2 k_{1} R\right),-\sin \left(2 k_{1} R\right)\right)$ and $\vec{M}_{k_{2}}(R) \propto k_{2}\left(\cos \left(2 k_{2} R\right)\right.$, $\left.\sin \left(2 k_{2} R\right)\right)$ of opposite vector chirality defined as $\vec{c}=$ $\vec{M}(R) \times \vec{M}(R+d R)$. The chirality $\vec{c}_{k_{2}}=\sin \left(2 k_{2} d R\right) \hat{e}_{\phi}$ points in the $\phi$ direction and forms a right-handed coordinate system with the directions $\hat{e}_{z}$ and $\hat{e}_{r}$, while $\vec{c}_{k_{1}}=$ $-\sin \left(2 k_{1} d R\right) \hat{e}_{\phi}$ points in the $-\phi$ direction and forms a left-handed coordinate system with $\hat{e}_{z}$ and $\hat{e}_{r}$. Accordingly, we call $\vec{M}_{k_{1}}$ and $\vec{M}_{k_{2}}$ a left- and right-rotating Skyrmionic wave, respectively. Our definition of a Skyrmion in the actual work designates well-defined magnetic waves which are (i) centered around the adatoms and have (ii) fixed rotation sense [1]. One great virtue of Skyrmions is their topological nature that is protected under the presence of reasonable size magnetic fields.

This multi-Skyrmionic magnetic waves can be destroyed or manipulated by modifying the spin-orbit interaction. By switching it off, only the magnetization along the $z$ direction would be finite. One way to tune the Rashba effect is to change the substrate nature gradually [31,32]. Such an experiment is very difficult to realize but we propose another tuning method: Instead of the Fermi energy, one could probe at different energy varying the bias voltage between tip and sample. For example, by decreasing the value of the energy probed, the wavelength corresponding to $2 k$ decreases which is accompanied by a decrease in the number of nodes. This is observable in

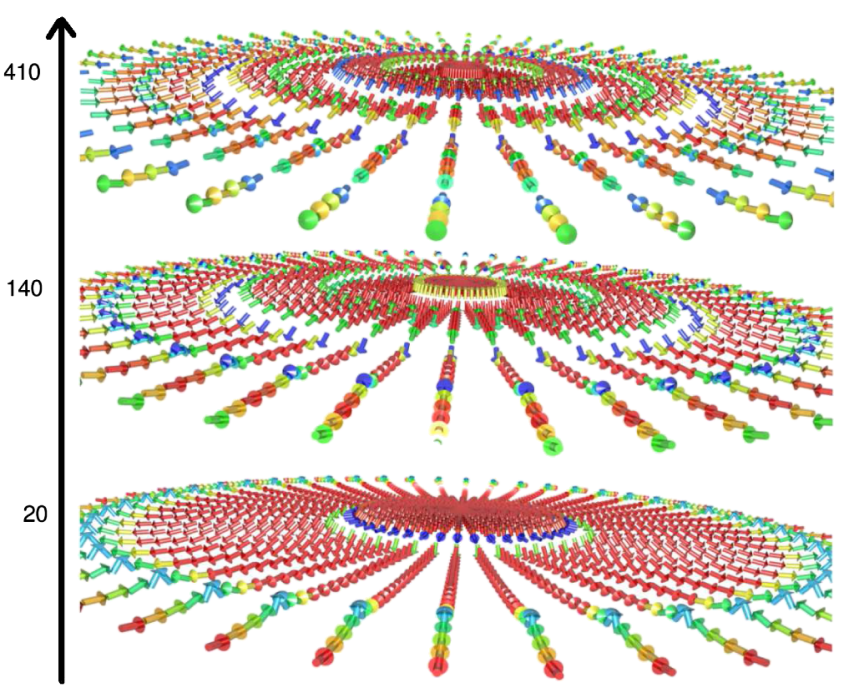

FIG. 4 (color online). Noncollinear spin configuration of the electron gas. Spectroscopic Skyrmionic-like spin textures of the electron magnetization surrounding the adatoms at different energies (410, 140, and $20 \mathrm{meV}$, respectively). The number of nodes diminishes when decreasing the energy, leading to smoother rotating spherical magnetic waves.

the two additional examples depicted in Fig. 3 calculated at 140 and $20 \mathrm{meV}$, respectively. The final multi-Skyrmionic waves have a different texture but share the common feature in the phase switch at $60 \AA$.

One should bear in mind that, as for the LDOS, two different regimes have to be expected for the magnetization components depending on the sign of $k_{2}$ (see Supplemental Material [30]). This suggests that the scanning tunneling spectroscopy (STS) experiment of Ast et al. [14], but spin polarized, would also be a way to verify our predictions.

To summarize, we have revealed a new type of spin texture (see Fig. 4) induced in a confined electron gas subject to the Rashba effect by a magnetic atom. These structures can be understood as a kind of combination of Skyrmionic-like waves of opposite chirality. By tuning the energy level or the spin-orbit strength, the observation of the final spin texture is possible because of the large magnitude of the induced magnetization and the creation and exploration of new complex configurations that are manageable with state-of-the-art experiments. Such nontrivial magnetic Friedel oscillations have an impact on the magnetic interactions between adatoms and other nanostructures and consequently on their magnetic behavior. Simple asymptotic expressions for the induced magnetizations are derived which offer a simple understanding of new types of experiments involving the manipulation and construction of adatom-based spin nanostructures.

We thank Professor K. Scharnberg for stimulating discussions at the initial stage of the work, and Dr. P. Lazic and Dr. H. Schumacher for their assistance in preparing some of the figures. S. L. acknowledges the support of the 
HGF-YIG Programme VH-NG-717 (Functional Nanoscale Structure and Probe Simulation Laboratory, Funsilab).

*s.lounis@fz-juelich.de

[1] U. K. Rößler, A. N. Bogdanov, and C. Pfleiderer, Nature (London) 442, 797 (2006); U. K. Rößler, A. A. Leonov, and A. N. Bogdanov, J. Phys. Conf. Ser. 303, 012105 (2011).

[2] S. Mühlbauer, B. Binz, F. Jonietz, A. Pfleiderer, A. Rosch, R. Neubauer, R. Georgii, and P. Böni, Science 323, 915 (2009).

[3] S. Heinze, K. von Bergmann, M. Menzel, J. Brede, A. Kubetzka, R. Wiesendanger, G. Bihlmayer, and S. Blügel, Nature Phys. 7, 713 (2011).

[4] M. Bode, M. Heide, K. von Bergmann, P. Ferriani, S. Heinze, G. Bihlmayer, A. Kubetzka, O. Pietzsch, S. Blügel, and R. Wiesendanger, Nature (London) 447, 190 (2007).

[5] Y.M. Koroteev, G. Bihlmayer, J.E. Gayone, E. V. Chulkov, S. Blügel, P. M. Echenique, and Ph. Hofmann, Phys. Rev. Lett. 93, 046403 (2004).

[6] C. R. Ast, J. Henk, A. Ernst, L. Moreschini, M. C. Falub, D. Pacilé, P. Bruno, K. Kern, and M. Grioni, Phys. Rev. Lett. 98, 186807 (2007).

[7] S. LaShell, B. A. McDougall, and E. Jensen, Phys. Rev. Lett. 77, 3419 (1996).

[8] M. Hasan and C. Kane, Rev. Mod. Phys. 82, 3045 (2010).

[9] R. R. Biswas and A. V. Balatsky, Phys. Rev. B 81, 233405 (2010).

[10] H.-M. Guo and M. Franz, Phys. Rev. B 81, 041102(R) (2010).

[11] F. Reinert, G. Nicolay, S. Schmidt, and D. Ehm, Phys. Rev. B 63, 115415 (2001).

[12] G. Nicolay, F. Reinert, S. Hüfner, and P. Blaha, Phys. Rev. B 65, 033407 (2001).

[13] J. Henk, A. Ernst, and P. Bruno, Phys. Rev. B 68, 165416 (2003).
[14] C. R. Ast, G. Wittich, P. Wahl, R. Vogelgesang, D. Pacilé, M. C. Falub, L. Moreschini, M. Papagno, M. Grioni, and K. Kern, Phys. Rev. B 75, 201401(R) (2007).

[15] A. Strozecka, A. Eiguren, and J. I. Pascual, Phys. Rev. Lett. 107, 186805 (2011)

[16] L. Petersen and P. Hedegård, Surf. Sci. 459, 49 (2000).

[17] J. D. Walls and E. J. Heller, Nano Lett. 7, 3377 (2007); J. D. Walls, J. Huang, R. M. Westervelt, and E. J. Heller, Phys. Rev. B 73, 035325 (2006).

[18] A. T. Ngo, J. Rodriguez-Laguna, S.E. Ulloa, and E. H. Kim, Nano Lett. 12, 13 (2012).

[19] T. H. Skyrme, Proc. R. Soc. A 260, 127 (1961).

[20] A. A. Belavin and A. M. Polyakov, JETP Lett. 22, 245 (1975).

[21] E. I. Rashba, Sov. Phys. Solid State 2, 1109 (1960).

[22] E. J. Heller, M. F. Crommie, C. P. Lutz, and D. M. Eigler, Nature (London) 369, 464 (1994); G. A. Fiete and E. J. Heller, Rev. Mod. Phys. 75, 934 (1993).

[23] M. F. Crommie, C. P. Lutz, and D. M. Eigler, Science 262, 218 (1993).

[24] H.C. Manoharan, C.P. Lutz, and D. M. Eigler, Nature (London) 403, 512 (2000); C. R. Moon, L. S. Mattos, B. K. Foster, G. Zeltzer, and H.C. Manoharan, Nature Nanotech. 4, 167 (2009);

[25] J. Tersoff and D. R. Hamann, Phys. Rev. Lett. 50, 1998 (1983).

[26] D. Wortmann, S. Heinze, Ph. Kurz, G. Bihlmayer, and S. Blügel, Phys. Rev. Lett. 86, 4132 (2001).

[27] T. Jamneala, V. Madhavan, W. Chen, and M. Crommie, Phys. Rev. B 61, 9990 (2000).

[28] J. Kondo, Prog. Theor. Phys. 32, 37 (1964).

[29] N. Papanikolaou, R. Zeller, and P. H. Dederichs, J. Phys. Condens. Matter 14, 2799 (2002); H. Ebert and R. Zeller, The SPR-TB-KKR package. http://olymp.cup.unimuenchen.de/ak/ebert/SPR-TB-KKR, 2002.

[30] See Supplemental Material at http://link.aps.org/ supplemental/10.1103/PhysRevLett.108.207202 for details.

[31] H. Asonen and M. Pessa, Phys. Rev. Lett. 46, 1696 (1981).

[32] S. A. Lindgren and L. Walldén, Surf. Sci. 89, 319 (1979). 